SMAD, Rev Eletrônica Saúde Mental Álcool Drog.

\title{
Associações entre uso de álcool e perfil sociodemográfico de universitários de Enfermagem e Nutrição
}

Bárbara Souza de Medeiros Nunes ${ }^{1}$

(D) https://orcid.org/0000-0002-6791-4523

Guilherme Correa Barbosa ${ }^{1}$

(D) https://orcid.org/0000-0002-7433-8237

Thiago da Silva Domingos ${ }^{1}$

(D) https://orcid.org/0000-0002-1421-7468
Universidade Estadual Paulista "Júlio de Mesquita Filho" UNESP, Faculdade de Medicina de Botucatu, Botucatu, SP, Brasil.
Objetivo: verificar o padrão de uso de álcool em graduandos dos cursos de enfermagem e nutrição de uma universidade pública e possíveis associações entre as variáveis sociodemográficas. Método: estudo transversal realizado com 170 estudantes dos cursos de graduação em Enfermagem e Nutrição de uma universidade pública. Utilizou-se um questionário sociodemográfico e o Alcohol Use Disorder Identification Test. As associações foram verificadas por meio do teste qui-quadrado considerando p-valor <0,05. Resultados: a prevalência de consumo foi de $80 \%$ e binge drinking, 59,53\%. O padrão de baixo risco foi identificado em $53,53 \%$ dos estudantes. Associaram-se aos perfis de consumo álcool a faixa etária atual e de primeiro contato com bebidas alcoólicas, composição domiciliar e tipo de bebida alcoólica consumida. Conclusão: aos padrões de riscos baixo e nocivo, entre estudantes de Enfermagem e Nutrição, associaram-se as variáveis faixa etária entre 17 e 23 anos, idade de primeiro contato com bebida alcoólica entre 10 e 17 anos, e residir com amigos. O uso de bebidas fermentadas associou-se ao padrão de baixo risco. 0 reconhecimento do perfil contribui para o desenvolvimento de estratégias de promoção, prevenção e educação em saúde a fim de reduzir os riscos futuros decorrentes do uso de álcool.

Descritores: Estudantes de Ciências da Saúde; Consumo de Álcool na Faculdade; Enfermagem; Recursos Humanos em Nutrição; Política Pública.

\section{Como citar este artigo}

Nunes BSM, Barbosa GC, Domingos TS. Associations between alcohol use and the sociodemographic profile of nursing and nutrition students. SMAD, Rev Eletrônica Saúde Mental Álcool Drog. 2021 abr.-jun.;17(2):72-81. doi: https://dx.doi.org/10.11606/issn.1806-6976.smad.2021.162031 


\section{Associations between alcohol use and the sociodemographic profile of nursing and nutrition students}

Objective: to verify the pattern of alcohol use in undergraduate nursing and nutrition students at a public university and possible associations between sociodemographic variables. Method: a cross-sectional study with 170 undergraduate Nursing and Nutrition students from a public university. A sociodemographic questionnaire and the Alcohol Use Disorder Identification Test were used for data collection. Associations were verified by the chi-square test considering a p-value $<0.05$. Results: the prevalence of consumption was $80 \%$, and of binge drinking, $59.53 \%$. The low risk pattern was identified in $53.53 \%$ of the students. Alcohol consumption profiles were associated with current age range and age of first contact with alcohol, household composition and type of alcohol consumed. Conclusion: among nursing and nutrition students, low and harmful risk patterns were associated with the following variables: age group between 17 and 23 years old, age of first contact with alcohol between 10 and 17 years, and living with friends. The use of fermented drinks was associated with the low risk pattern. The recognition of the profile contributes to the development of health promotion, prevention and education strategies in order to reduce future risks arising from the use of alcohol.

Descriptors: Students, Health Occupations; Alcohol Drinking in College; Nursing; Nutrition Personnel; Public Policy.

\section{Asociaciones entre el consumo de alcohol y el perfil sociodemográfico de los estudiantes de enfermería y nutrición}

Objetivo: verificar el patrón de consumo de alcohol en estudiantes de licenciatura en enfermería y nutrición de una universidad pública y sus posibles asociaciones entre variables sociodemográficas. Método: estudio transversal con 170 estudiantes de pregrado de Enfermería y Nutrición de una universidad pública. Para la recopilación de datos se utilizó un cuestionario sociodemográfico y el Test de identificación de los trastornos debidos al consumo de alcohol (Alcohol Use Disorder Identification Test -AUDIT). Las asociaciones se verificaron mediante prueba de chi cuadrado considerando valor $p<0,05$. Resultados: la prevalencia de consumo fue del $80 \%$ y el consumo excesivo de alcohol del 59,53\%. El patrón de bajo riesgo se identificó en $53,53 \%$ de los estudiantes. Los perfiles de consumo de alcohol se asociaron con la edad actual y el primer contacto con el alcohol, la composición del hogar y el tipo de alcohol consumido. Conclusión: a los patrones de riesgo bajo y nocivo, entre los estudiantes de Enfermería y Nutrición, se asociaron las variables grupo de edad entre 17 y 23 años, edad de primer contacto con alcohol entre 10 y 17 años y convivencia con amigos. El uso de bebidas fermentadas se asoció con el patrón de bajo riesgo. El reconocimiento del perfil contribuye al desarrollo de estrategias de promoción, prevención y educación de la salud con el fin de reducir los riesgos futuros derivados del consumo de alcohol.

Descritores: Estudiantes del Área de la Salud; Consumo de Alcohol en la Universidad; Enfermería; Personal de Nutrición; Política Pública. 


\section{Introdução}

A Organização Mundial da Saúde (OMS) afirma que cerca de 2,3 bilhões de pessoas são usuários de álcool, sendo consumido por mais da metade da população das Américas, da Europa e do Pacífico Ocidental, e resultando em aproximadamente três milhões de mortes por ano, o que corresponde a um percentual de $5,3 \%$ do total de mortes ${ }^{(1)}$.

No Brasil, observou-se que $16 \%$ da população fazem uso nocivo de álcool e cerca de $50 \%$ o fizeram uso nos últimos 12 meses, deste montante, 62\% correspondem a pessoas do sexo masculino. Esses dados indicam que o uso de álcool vem ocorrendo em faixas etárias cada vez mais precoces, contexto que aponta para a necessidade de revisão das medidas de controle, prevenção e tratamento(2).

O I Levantamento Nacional sobre o Uso de Álcool, Tabaco e Outras Drogas entre Universitários das 27 capitais brasileiras apontou que $86,5 \%$ da população universitária já experimentaram álcool alguma vez na vida, 60,5\% declararam ter feito uso de álcool nos últimos 30 dias, 19,2\% dos universitários preencheram os critérios para uso abusivo, e 2,6\%, os critérios para uso de alto risco ou de dependência(3). Assim, o uso de álcool representa a substância psicoativa mais utilizada entre os universitários ${ }^{(4-5)}$ e cuja prevalência é maior em comparação com a população em geral $(89,3 \%$ versus $78,6 \%)^{(6)}$.

Nesse sentido, explora-se o questionamento acerca da vida universitária atuar como fator de proteção ou de risco para o uso de álcool e outras drogas( ${ }^{(4-5,7-9)}$, uma vez que vêm sendo utilizadas pelos estudantes também como estratégia de lazer(10).

Estudos apresentam o álcool como a substância lícita mais utilizada entre a população universitária independentemente do curso ou do recorte temporal investigado que, geralmente, varia entre os últimos 30 dias e três meses, tendo uma tendência de a prevalência do uso de álcool ser maior à medida que decorre os anos do curso de graduação. Apontam também que universitários com mais sintomas de depressão e ansiedade relataram maiores aumentos de consumo álcool em comparação com alunos com menos sintomas ${ }^{(11-14)}$.

Divergências em relação às características sociodemográficas de estudantes universitários e o uso de álcool podem ser identificados entre as investigações que se debruçam sobre a temática. Há resultados indicando que estudantes de enfermagem do sexo feminino apresentam maior prevalência de uso álcool quando comparada à população do sexo masculino(7). Contudo, quando se amplia o escopo de análise incluindo diversos cursos de nível superior, o sexo masculino surge como uma variável associada positivamente ao envolvimento com substâncias lícitas e ilícitas ${ }^{(9)}$, mas o sexo feminino indica associação ao risco moderado para desenvolver dependência( ${ }^{(8)}$.

Além desse contexto, dados apresentados pelo Censo da Educação Superior permitem afirmar que a população de estudantes universitários brasileiros ultrapassa sete milhões, tendo aumento $17 \%$ entre os anos de 2010 e 2013 nas Instituições de Ensino Superior de categoria pública(15). Assim, reconhecer e ampliar a influência das variáveis que interferem sobre o uso de álcool entre estudantes universitários das ciências da saúde é fundamental para o desenvolvimento de estratégias de prevenção, promoção e cuidado efetivas, voltadas diretamente para esse segmento da população.

Compreender o uso de álcool como um problema de saúde pública significa tomá-la e abordá-la em sua multifatoriedade e dinamicidade. As imbricadas dimensões psíquicas, orgânicas, socioeconômicas e histórico-culturais em constante modificação representam desafios para constituição de estratégias e políticas para responder às necessidades de saúde(16).

Com a finalidade de elucidar as relações entre as variáveis sociodemográficas e o uso de álcool entre estudantes de ciências da saúde, partiu-se da seleção de dois cursos que, como particularidade conta com a predominância de estudantes do sexo feminino, para responder a seguinte pergunta de pesquisa: "Como se caracteriza o padrão uso de álcool de graduandos de uma universidade pública do interior do estado de São Paulo e quais as associações entre as variáveis sociodemográficas dessa população?"

Pelo exposto, objetivou-se, por meio dessa investigação, verificar o padrão de uso de álcool em graduandos dos cursos de enfermagem e nutrição de uma universidade pública e possíveis associações entre as variáveis sociodemográficas.

\section{Método}

Trata-se de um estudo transversal, do tipo descritivoexploratório e de caráter quantitativo. Foi realizado em uma universidade pública situada no interior do estado de São Paulo. De um universo de 240 estudantes matriculados nos cursos de Enfermagem e Nutrição, participaram 170 , quantidade que superou a quantidade proposta pelo cálculo amostral. A distribuição entre os cursos foi, respectivamente, 91 e 79 estudantes matriculados do primeiro ao último ano de cada curso, tendo como critério de inclusão estar regularmente matriculado em um dos dois cursos no ano 2016.

Os dados foram coletados durante os meses de março a maio de 2016. Utilizaram-se como instrumentos de coleta de dados um questionário sociodemográfico e um questionário autoaplicável denominado Alcohol Use Disorder Identification Test (AUDIT) para verificar o padrão do consumo de álcool. O AUDIT é composto por 
10 questões objetivas que permitem respostas com pesos preestabelecidos, variando de zero a quatro. O somatório do peso de cada questão indica a classificação de cada indivíduo frente ao padrão consumo de álcool, como se segue: zero a sete padrão de beber moderado, oito a 15 beber de risco, de 16 a 19 uso nocivo de álcool, e de 20 a 40 possível dependência de álcool(17-18).

Para operacionalizar a coleta, foi solicitada a permissão ao docente de uma determinada disciplina para aplicação do instrumento de coleta. O docente indicou o início ou final da aula como o melhor momento para a coleta. Realizou-se o contato com os estudantes em sala com a finalidade de apresentar a proposta da pesquisa e solicitar a assinatura no Termo de Consentimento Livre e Esclarecido (TCLE) de cada estudante. Após essa etapa, foi realizada a distribuição dos instrumentos de coleta dos dados e recolhidos ao término do preenchimento.

As respostas foram organizadas em um banco de dados utilizando programa de computador Excel (versão 2007) e para a análise estatística dos dados, o sistema Statistical Analysis Software (SAS). Para verificar as associações entre as variáveis sociodemográficas e zonas do AUDIT foi utilizado o teste qui-quadrado considerando Intervalo de Confiança (IC) $95 \%$ e valor de $p<0,05$. Os resultados foram reunidos e apresentados em tabelas com suas respectivas frequências absolutas e relativas, e $p$-valor.

O projeto foi submetido ao Comitê de Ética em Pesquisa da Faculdade de Medicina de Botucatu da Universidade Estadual "Júlio de Mesquita Filho", seguindo as determinações da Resolução 466/12, do Conselho Nacional de Ética em Pesquisa - CONEP/CNS e aprovado sob o parecer de no 1.048 .615 .

\section{Resultados}

Os dados a seguir correspondem ao perfil sociodemográfico, nota-se na população de estudo predomínio do sexo feminino, da cor branca, sem parceiro, residindo com amigos/república estudantil. A média etária foi 21 anos [Desvio Padrão (DP)=2,97], variando entre 18 e 27 anos. No que se refere à procedência, 95,88\% ( $n=163$ ) eram do Estado de São Paulo, sendo que 14,71\% $(n=25)$ pertenciam à cidade de Botucatu. A maioria não declarou vínculo empregatício $(93,49 \%, n=158)$.

Tabela 1 - Distribuição das Frequências e respostas $(n=170)$. Botucatu, SP, Brasil, 2016

\begin{tabular}{|c|c|c|}
\hline Variáveis & $\mathbf{n}$ & $\%$ \\
\hline \multicolumn{3}{|l|}{ Gênero } \\
\hline Feminino & 153 & 90,00 \\
\hline Masculino & 17 & 10,00 \\
\hline \multicolumn{3}{|l|}{ Cor da pele } \\
\hline Branca & 129 & 75,88 \\
\hline
\end{tabular}

\begin{tabular}{|c|c|c|}
\hline Variáveis & $\mathrm{n}$ & $\%$ \\
\hline Não branca & 41 & 24,12 \\
\hline \multicolumn{3}{|l|}{ Estado civil } \\
\hline Sem parceiro & 164 & 96,47 \\
\hline Com parceiro & 6 & 3,53 \\
\hline \multicolumn{3}{|l|}{ Vínculo empregatício } \\
\hline Sim & 11 & 6,51 \\
\hline Não & 158 & 93,49 \\
\hline \multicolumn{3}{|l|}{ Composição domiciliar } \\
\hline República estudantil/amigos & 102 & 60,00 \\
\hline Família & 35 & 20,59 \\
\hline Sozinho & 33 & 19,41 \\
\hline \multicolumn{3}{|l|}{ Curso } \\
\hline Enfermagem & 91 & 53,53 \\
\hline Nutrição & 79 & 46,47 \\
\hline \multicolumn{3}{|l|}{ Ano } \\
\hline $1^{\circ}$ & 53 & 31,18 \\
\hline $2^{\circ}$ & 33 & 19,41 \\
\hline $3^{\circ}$ & 50 & 29,41 \\
\hline $4^{\circ}$ & 34 & 20,00 \\
\hline
\end{tabular}

A iniciação do uso do álcool ocorreu na média etária de 15 anos ( $31,18 \%, n=53)$, variando entre 10 e 25 anos, sendo que a maioria $(77,05 \%, n=131)$ não percebeu 0 consumo de bebida alcoólica como um problema. O tipo de bebida alcoólica mais consumida foi a cerveja (49,41\%, $\mathrm{n}=84)$, seguida dos destilados $(35,29 \%, \mathrm{n}=60)$.

Ao observar o padrão de consumo, $13,53 \%(n=23)$ referiram nunca ter feito consumo de álcool na vida. Neste caminho, 59,41\% $(n=101)$ revelaram ter consumido cinco ou mais doses de álcool em uma única ocasião (binge drinking). Considerando a frequência dos episódios de binge drinking, 28,82\% $(n=49)$ foi mensal e $15,29 \%$ $(n=26)$, semanalmente.

No que se refere aos sintomas de dependência, observou-se que $15,88 \%(n=27)$ dos participantes revelaram a percepção de não conseguir parar de beber após terem iniciado; destes, $4,12 \%(n=7)$ tiveram esta percepção mensalmente e $1,18 \%(n=2)$ semanalmente. Observou-se que $37,65 \%(n=64)$ já haviam deixado de fazer o que era esperado devido ao uso de álcool. Além disso, 2,35\% $(n=4)$ revelaram ter precisado de uma primeira dose pela manhã para sentirem-se melhor depois de uma bebedeira.

Quanto aos problemas decorrentes do uso de álcool, constatou-se que $44,11 \%(n=75)$ dos universitários já sentiram culpa ou remorso depois de beber, sendo que $7,65 \%(n=13)$ arrependeram-se mensal ou semanalmente por ingerir bebida alcoólica. Apagamentos (blackouts) ocorreram em $56,47 \%(n=96)$ da população de estudo, com frequência mensal em 7,06\% $(n=12)$, e semanal 0,59\% $(n=1)$. 
Uma parcela da população de estudo $(24,12 \%, n=41)$ recebeu críticas de terceiros em relação aos resultados negativos devido ao uso de álcool. Uma parcela menor $(12,94 \%, n=12)$ foram aconselhados a parar de beber.

O padrão do uso de álcool está apresentado na Tabela 2. Os resultados evidenciaram uma maioria de estudantes $(53,53 \%, n=91)$ apresentando um padrão de uso de baixo risco.

Tabela 2 - Padrão do consumo de álcool entre os estudantes universitários ( $n=170)$, Botucatu, SP, Brasil, 2016

\begin{tabular}{lcc}
\hline Perfis de Consumo & $\boldsymbol{n}$ & $\%$ \\
\hline Baixo Risco & 91 & 53,53 \\
Uso de Risco & 59 & 34,71 \\
Uso Nocivo & 18 & 10,59 \\
Provável Dependência & 2 & 1,18 \\
\hline
\end{tabular}

Entre as quatro zonas do perfil de consumo propostas pelo AUDIT, baixo risco, uso de risco, uso nocivo e provável dependência, foram verificadas possíveis associações com as características sociodemográficas, conforme apresenta a Tabela 3. Observa-se que a faixa etária entre 18 e 23 anos, residir com amigos, ter tido o primeiro contato com bebida alcoólica entre 10 e 17 anos foram dados que se associaram aos perfis de consumo de baixo risco e uso nocivo. O uso de bebidas fermentadas se associou apenas ao perfil de consumo de baixo risco. Ressalta-se que mesmo diante da predominância do sexo feminino, essa variável não se associou ao perfil de consumo.

Tabela 3 - Associações entre características sociodemográficas e perfis de consumo do AUDIT* $(n=170)$. Botucatu, SP, Brasil, 2016

\begin{tabular}{|c|c|c|c|c|c|c|c|c|c|}
\hline \multirow{3}{*}{$\begin{array}{l}\text { Características } \\
\text { sociodemográficas }\end{array}$} & \multicolumn{8}{|c|}{ Perfis de Consumo (AUDIT*) } & \multirow{3}{*}{ p-value } \\
\hline & \multicolumn{2}{|c|}{ I } & \multicolumn{2}{|c|}{ II } & \multicolumn{2}{|c|}{ III } & \multicolumn{2}{|c|}{ IV } & \\
\hline & $n$ & $\%$ & $n$ & $\%$ & $n$ & $\%$ & $n$ & $\%$ & \\
\hline \multicolumn{10}{|l|}{ Sexo } \\
\hline Feminino & 11 & 6,47 & 4 & 2,35 & 2 & 1,18 & 0 & 0 & \multirow{2}{*}{, 7132} \\
\hline Masculino & 80 & 47,06 & 55 & 32,55 & 16 & 9,41 & 2 & 1,18 & \\
\hline \multicolumn{10}{|l|}{ Cor } \\
\hline Branca & 69 & 40,59 & 47 & 27,65 & 12 & 7,06 & 1 &, 59 & \multirow{2}{*}{, 5666} \\
\hline Não Branca & 22 & 12,94 & 12 & 7,06 & 6 & 3,53 & 1 &, 59 & \\
\hline \multicolumn{10}{|l|}{ Faixa etária (anos) } \\
\hline $18-20$ & 38 & 22,35 & 40 & 23,35 & 6 & 3,53 & 1 &, 59 & \multirow{3}{*}{,0079 } \\
\hline $22-23$ & 39 & 22,94 & 19 & 11,18 & 10 & 5,88 & 1 &, 59 & \\
\hline$\geq 24$ & 14 & 8,24 & 0 & 0 & 2 & 1,18 & 0 & 0 & \\
\hline \multicolumn{10}{|l|}{ Estado civil } \\
\hline Com parceiro & 87 & 51,18 & 57 & 33,53 & 18 & 10,59 & 2 & 1,18 & \multirow{2}{*}{,9349 } \\
\hline Sem parceiro & 4 & 2,35 & 2 & 1,18 & 0 & 0 & 0 & 0 & \\
\hline \multicolumn{10}{|l|}{ Procedência } \\
\hline Outro município & 73 & 42,94 & 54 & 31,76 & 16 & 9,41 & 2 & 1,18 & \multirow{2}{*}{,2382 } \\
\hline Mesmo município & 18 & 10,59 & 5 & 2,94 & 2 & 1,18 & 0 & 0 & \\
\hline \multicolumn{10}{|l|}{ Vínculo empregatício } \\
\hline Sim & 8 & 4,73 & 3 & 1,78 & 0 & 0 & 0 & 0 & \multirow{2}{*}{,4887 } \\
\hline Não & 82 & 48,52 & 56 & 33,14 & 18 & 10,65 & 2 & 1,18 & \\
\hline \multicolumn{10}{|l|}{ Composição domiciliar } \\
\hline Sozinho & 23 & 13,53 & 9 & 5,29 & 1 &, 59 & 0 & 0 & \multirow{3}{*}{,0322 } \\
\hline Família & 24 & 14,12 & 8 & 4,71 & 2 & 1,18 & 1 &, 59 & \\
\hline Amigos & 44 & 25,88 & 42 & 24,71 & 15 & 8,82 & 1 &, 59 & \\
\hline \multicolumn{10}{|l|}{ Cursos } \\
\hline Enfermagem & 54 & 31,76 & 27 & 15,88 & 8 & 4,71 & 2 & 1,18 & \multirow{2}{*}{,1718 } \\
\hline Nutrição & 37 & 21,76 & 32 & 18,82 & 10 & 5,88 & 0 & 0 & \\
\hline
\end{tabular}




\begin{tabular}{|c|c|c|c|c|c|c|c|c|c|}
\hline \multirow{3}{*}{$\begin{array}{l}\text { Características } \\
\text { sociodemográficas }\end{array}$} & \multicolumn{8}{|c|}{ Perfis de Consumo (AUDIT*) } & \multirow{3}{*}{ p-value } \\
\hline & \multicolumn{2}{|c|}{1} & \multicolumn{2}{|c|}{ II } & \multicolumn{2}{|c|}{ III } & \multicolumn{2}{|c|}{ IV } & \\
\hline & $n$ & $\%$ & $n$ & $\%$ & $n$ & $\%$ & $n$ & $\%$ & \\
\hline \multicolumn{10}{|l|}{ Ano dos Cursos } \\
\hline 1 & 28 & 16,47 & 22 & 12,94 & 2 & 1,18 & 1 &, 59 & \multirow{4}{*}{,1153 } \\
\hline 2 & 18 & 10,59 & 14 & 8,24 & 1 &, 59 & 0 & 0 & \\
\hline 3 & 26 & 15,29 & 16 & 9,41 & 8 & 4,71 & 0 & 0 & \\
\hline 4 & 19 & 11,18 & 7 & 4,12 & 7 & 4,12 & 1 &, 59 & \\
\hline \multicolumn{10}{|c|}{ Faixa etária (anos) do primeiro contato com bebida alcoólica } \\
\hline Sem contato & 5 & 2,94 & 0 & 0 & 0 & 0 & 0 & 0 & \multirow{5}{*}{,0105 } \\
\hline $10-14$ & 20 & 11,76 & 30 & 17,65 & 9 & 5,29 & 1 &, 59 & \\
\hline $15-17$ & 50 & 29,41 & 28 & 16,47 & 5 & 2,94 & 1 &, 59 & \\
\hline $18-20$ & 15 & 8,82 & 1 &, 59 & 4 & 2,35 & 0 & 0 & \\
\hline$\geq 21$ & 1 &, 59 & 0 & 0 & 0 & 0 & 0 & 0 & \\
\hline \multicolumn{10}{|c|}{ Tipo de bebida alcoólica } \\
\hline Sem contato & 21 & 12,35 & 2 & 1,18 & 0 & 0 & 0 & 0 & \multirow{4}{*}{0221} \\
\hline Destilada & 27 & 15,88 & 26 & 15,29 & 7 & 4,12 & 0 & 0 & \\
\hline Fermentada & 42 & 24,71 & 29 & 17,06 & 11 & 6,47 & 2 & 1,18 & \\
\hline Ambas & 1 &, 59 & 2 & 1,18 & 0 & 0 & 0 & 0 & \\
\hline
\end{tabular}

*AUDIT $=$ Alcohol Use Disorder Identification Test

\section{Discussão}

A prevalência de uso de álcool na população investigada foi de $80 \%$ e prática do binge drinking, 59,41\%. Esses índices se aproximam de uma pesquisa realizada na região sul do Brasil, envolvendo 12 cursos da área da saúde que encontrou, respectivamente, prevalências de $85 \%$ e $56,1 \%{ }^{(19)}$. Populações semelhantes em outras regiões do país e da América Latina apresentaram prevalência aproximada de consumo $(74,9 \%)^{(20)},(83,7 \%)^{(7)},(85 \%)^{(21)}$ e $(86 \%)^{(22)}$.

Contudo, entre estudantes de medicina de instituição particular brasileira a prevalência do uso foi menor $(69,3 \%)^{(23)}$, assim como, em instituições peruanas que indicou 48,5\% ${ }^{(24)}$. Uma análise de estudantes universitários brasileiros e portugueses apontou que no tempo livre $56,8 \%$ fazem uso de álcool e 43,5\%, binge drinking(25).

Observa-se uma variação na prevalência do uso de álcool e de binge drinking de acordo com a população e o contexto. Entre estudantes franceses, a prática de binge drinking atingiu a prevalência de $65,1 \%{ }^{(26)}$, enquanto que no cenário nacional, a prevalência de binge drinking foi aproximadamente quatro vezes menor $(15,3 \%)^{(20)}$ àquela comparada ao resultado desse estudo. Os resultados dessa investigação foram maiores aos encontrados entre estudantes das área de exatas $(34,7 \%)$ e biológicas $(24,6 \%)^{(3)}$.

Sabe-se que o binge drinking está associada a consequências negativas, como: baixo rendimento escolar e acidentes ${ }^{(3)}$. Aponta-se que há, entre os estudantes de Nutrição e Enfermagem, uma parcela maior cujo padrão de consumo pode acarretar em repercussões negativas, situação que se agrava quando se observa a alta prevalência de binge drinking. Um amplo estudo francês observou que a prática de binge drinking esteve associado ao consumo de maconha e tabaco(26).

Essa prática associou-se também a comportamentos de riscos, entre os quais: dirigir sob o efeito do álcool, pegar carona com alguém que consumiu bebida alcoólica, envolvimento em acidentes de trânsito, problemas acadêmicos, problemas com relacionamentos afetivos, envolvimento em brigas e infrações legais, prática sexual sem uso de preservativo, náuseas e vômitos(19,27-29).

Verificou-se que a prática de binge drinking foi maior entre estudantes do sexo masculino, aqueles que não declararam prática religiosa ou prática diferente do catolicismo, que estiveram envolvidos em brigas, que se ausentaram das atividades e que apresentaram baixo desempenho acadêmico(3,20).

A população dessa pesquisa classificada no perfil de consumo de baixo risco $(53,53 \%)$ foi menor quando comparada a outras investigações envolvendo estudantes de cursos da saúde $(77 \%)^{(19)}$, com estudantes do curso de medicina de instituição privada $(64 \%)^{(26)}$ e com estudantes de diversos cursos superiores $(77,5 \%)^{(30)}$, $(81 \%)^{(8)}$. Contudo, pesquisa realizada especificamente com estudantes de Enfermagem aponta uma porcentagem ainda menor $(21 \%)^{(28)}$ da encontrada nesse estudo.

Entre as variáveis associadas ao perfil de consumo, os resultados dessa pesquisa marcaram o predomínio do 
sexo feminino (90\%) como uma variável de proteção, por outro lado, identificaram-se como fatores de exposição estudantes que se declararam sem parceiros $(96,47 \%)$, viverem com amigos (60\%) e poucos morarem com os pais (20,59\%). Discute-se que residir com os pais, não ter iniciado vida sexual e prática religiosa foram três variáveis identificadas como fatores protetivos para o uso de álcool na faculdade ${ }^{(19,23,27)}$.

Uma parcela de estudantes vivencia, durante o ingresso na universidade, o afastamento das relações familiares e sociais, a ligação com novas amizades e a adaptação à rotina inerente a demanda universitária. Fatores esses que podem aumentar a vulnerabilidade para o uso do álcool e outras drogas, bem como, desempenhar fator fundamental para a dependência(23-24).

Em contrapartida, vida sexual ativa, relacionamento estável, história familiar positiva para uso de álcool e transtorno mental, tratamento prévio para transtorno mental, o convívio com amigos que usam álcool e frequentar festas uma ou mais vezes semanalmente foram fatores de exposição(19,23,30). Há divergências entre o estado civil, uma vez que estar solteiro foi uma variável de exposição em outras investigações ${ }^{(19-29)}$.

A maior prevalência do padrão do uso de álcool de baixo risco entre os universitários entrevistados pode estar relacionada aos cursos da área da saúde que, tradicionalmente, atrai mais mulheres do que homens. Todavia, não houve associação entre o sexo dos participantes e os perfis de consumo do AUDIT. Mas, deve-se observar uma mudança na dinâmica da distribuição do sexo e do gênero em relação ao padrão de uso de álcool entre universitários dos cursos de ciências da saúde, uma vez que estudos têm apontado um aumento na prevalência de estudantes do sexo feminino ${ }^{(5,7,13)}$.

Ao analisar o padrão do uso de álcool entre os gêneros, observa-se que o sexo masculino aparece com uma menor proporção de bebedores de baixo risco e maior índice de binge drinking frequente, o perfil oposto é característico do sexo feminino: maior proporção do padrão baixo risco e menor frequência de binge drinking (26-27,31-32).

Em um estudo multicêntrico realizado no Peru entre estudantes de medicina, o sexo masculino apresentou o dobro da frequência de consumo que o sexo feminino, além disso, associou positivamente ao perfil de dependência(20). No Brasil, o sexo masculino também esteve associado ao consumo de álcool em algum momento da vida de estudantes universitários ${ }^{(33)}$.

O presente estudo evidenciou que 147 (86,47\%) dos entrevistados tinham o hábito de ingerir álcool cuja idade de iniciação do uso ocorreu na faixa etária dos 10 aos 25 anos, característica associada ao perfil de consumo do AUDIT. Observa-se que em comparação a outros estudos, trata-se de uma faixa etária mais ampla ${ }^{(7,20,29,32,34)}$ e que inclui o período de ingresso no ensino superior, situação que indica a necessidade de estratégias preventivas e promotoras de atitudes responsáveis em torno do uso de álcool.

No quesito do tipo de bebida alcoólica mais consumida, identifica-se maior prevalência para uso da cerveja, característica que se alinha a outras pesquisas ${ }^{(7,25,29)}$ e também se associou aos perfis de consumo do AUDIT. Argumenta-se acerca do fácil acesso a esses tipos de bebidas, além de serem encontrados em todos os lugares e a preços baixos ${ }^{(4,19-20)}$.

Os fatores discutidos anteriormente refletem sobre as características sociodemográficas observadas nesta pesquisa e sustentam uma argumentação a favor da promoção e da prevenção. Nesse sentido, a educação para o uso do álcool envolvendo docentes e discentes contribui para desenvolver criticidade e autonomia acerca do uso consciente da bebida alcoólica, bem como, sensibilização para deteç̧ão precoce de problemas decorrentes do uso abusivo ou nocivo. Estudos que indicam pouca modificação no nível de conhecimento dos estudantes entre o início e o término do curso ${ }^{(30)}$ e essa problemática ser uma área de tensionamentos ainda permeada por silenciamentos institucionais ${ }^{(31)}$.

Os resultados dessa pesquisa permitiram identificar as variáveis sociodemográficas que influenciam o padrão de uso de álcool entre estudantes de Enfermagem e Nutrição. Evidenciou-se que a faixa etária entre 18 e 23 anos, residir com amigos e ter tido o primeiro contato com bebida alcoólica entre 10 e 17 anos foram aquelas que se associaram aos padrões de consumo de riscos baixo e nocivo. Enfatiza-se a alta prevalência do uso de álcool, da prática de binge drinking e da frequência de estudantes que relataram repercussões negativas devido ao uso de álcool.

A contribuição para a área da saúde e sua interface com a educação está no fato de que o padrão de uso de álcool entre jovens universitários constitui-se uma ferramenta apropriada para as instituições de ensino superior. Seu uso possibilita planejar intervenções preventivas estratégicas visando à redução dos riscos comportamentais e de saúde associados a essa problemática entre os estudantes, bem como, aumentando o nível de conhecimento dos estudantes no decorrer da vida universitária. Além disso, esse estudo soma-se a outros que visam caracterizar e discutir o perfil epidemiológico do consumo de álcool entre a população de estudantes universitários.

As limitações desse estudo se referem à restrição da amostra a dois cursos da área da saúde e à coleta de dados realizada no ano de 2016. Essas considerações, contudo, não fragilizam o diálogo dos resultados encontrados nessa investigação aos de outras pesquisas desenvolvidas em contexto nacional e internacional, 
fato que indica a contemporaneidade do problema de pesquisa e auxilia no direcionamento das políticas públicas.

\section{Conclusão}

Diante dos resultados desta pesquisa, detectou-se que entre estudantes de Enfermagem e Nutrição há predomínio do padrão de baixo risco para o uso de álcool, cuja iniciação ocorreu em uma faixa etária que antecede o ingresso no ensino superior. Verificou-se que as bebidas alcoólicas do tipo fermentadas são mais consumidas por essa população que se caracteriza também pelo predomínio do sexo feminino, estado civil solteiro e morando em repúblicas estudantis. Entre as associações detectadas entre os perfis de consumo de riscos baixo e nocivo estão as variáveis sociodemográficas: faixa etária 18 e 23 anos, primeiro contato com bebidas alcoólicas entre os 10 e 17 anos, e residir com amigos. O uso de bebida alcoólica fermentada foi mais frequente entre a perfil de baixo risco.

Assim, o delineamento do perfil de estudantes e do padrão de uso contribuem para o reconhecimento de variáveis de exposição e proteção, possibilitando o desenvolvimento de estratégias assertivas no que se refere à educação e promoção da saúde, bem como, a prevenção de problemas e agravos relacionados ao uso e abuso de álcool, reduzindo-se riscos futuros.

\section{Referências}

1. World Health Organization. Global Status Report on Alcohol and Health. [Internet]. 2018. [cited 2020 Aug 6]. Available from: https://www.who.int/substance_abuse/ publications/global_alcohol_report/gsr_2018/en/

2. Laranjeiras R, Madruga CS, Pinsky I, Caetano R, Mitsuhiro SS. II Levantamento Nacional de Álcool e Drogas (LENAD) - 2012. [Internet]. São Paulo: Instituto Nacional de Ciência e Tecnologia para Políticas Públicas de Álcool e Outras Drogas (INPAD), UNIFESP; 2014. [cited 2020 Aug 6]. Available from: https://inpad.org.br/wp-content/ uploads/2014/03/Lenad-II-Relat\%C3\%B3rio.pdf

3. Andrade A, Duarte $P$, Oliveira L. I Levantamento Nacional sobre o Uso de Álcool, Tabaco e Outras Drogas entre Universitários das 27 Capitais Brasileiras. [Internet]. Brasília: SENAD; 2010. [Acesso 12 jan 2018]. Disponível em: https://goo.gl/XMoVz7

4. Zanetti ACG, Cumsille F, Mann R. The association between the use of alcohol, marijuana and cocaine and the sociodemographic characteristics of university students of Ribeirão Preto, Brazil. Texto Contexto Enferm. 2019;28(spe):e110. doi: https://dx.doi. org/10.1590/1980-265x-tce-cicad-1-10

5. Santos DDM, Guimarães MM, Bodevan EC, Rocha RL, Pinheiro MLP. Use of psychoactive substances among university students. SMAD, Rev Eletrônica Saúde Mental Álcool Drog. 2019;15(3):1-9. doi: https://dx.doi. org/10.11606/issn.1806-6976.smad.2019.148973

6. Eckschmidt F, Andrade AG, Oliveira LG. Comparação do uso de drogas entre universitários brasileiros, norteamericanos e jovens da população geral brasileira. J Bras Psiquiatr. 2013 Set;62(3):199-207. doi: http://dx.doi. org/10.1590/S0047-20852013000300004

7. Rodríguez-Muñoz PM, Carmona-Torres JM, RodríguezBorrego MA. Influence of tobacco, alcohol consumption, eating habits and physical activity in nursing students. Rev. Latino-Am. Enfermagem. 2020;28:e3230. doi: http://dx.doi.org/10.1590/1518-8345.3198.3230

8. Silva DA, Pereira RJ Junior, Gomes CFM, Cardoso JV. Envolvimento com álcool, tabaco e outras substâncias por estudantes universitários. Rev Cuidarte. 2019;10(2):e641. doi: http://dx.doi.org/10.15649/cuidarte.v10i2.641

9. Granja GL, Lacerda-Santos JT, Brilhante DM, Nóbrega IS, Granville-Garcia AF, Caldas AF Júnior, et al. Smoking and alcohol consumption among university students of the healthcare area. J Public Health. (Berlin). 2020;28:4552. doi: https://doi.org/10.1007/s10389-018-01011-x 10. Vieira JL, Romera LA, Lima MCP. Lazer entre universitários da área da saúde: revisão de literatura. Ciênc Saúde Coletiva. 2018 Dec;23(12):4221-9. https:// doi.org/10.1590/1413-812320182312.31012016

11. Freitas CB, Veloso TCP, Segundo LPS, Sousa FPG, Galvão BS, Paixão PAR. Consumption of licit and illicit drugs by university students. Res Soc Develop. 2020;9(4):e189943016. doi: https://doi.org/10.33448/ rsd-v9i4.3016

12. Lechner WV, Laurene KR, Patel S, Anderson M, Grega $C$, Kenne DR. Changes in alcohol use as a function of psychological distress and social support following COVID19 related University closings. Addict Behav. 2020;10. doi: 10.1016/j.addbeh.2020.106527

13. Davoren MP, Demant J, Shiely F, Perry IJ. Alcohol consumption among university students in Ireland and the United Kingdom from 2002 to 2014: a systematic review. BMC Public Health. 2016;16:173. doi: https:// doi.org/10.1186/s12889-016-2843-1

14. Pacheco JP, Giacomin HT, Tam WW, Ribeiro TB, Arab C, Bezerra IM, et al. Mental health problems among medical students in Brazil: a systematic review and meta-analysis. Rev Bras Psiquiatr. 2017 Dec;39(4):369-78. doi: https:// doi.org/10.1590/1516-4446-2017-2223

15. Instituto Nacional de Estudos e Pesquisas Educacionais Anísio Teixeira (BR). Censo da educação superior 2013: resumo técnico. [Internet]. Brasília: Instituto Nacional de Estudos e Pesquisas Educacionais Anísio Teixeira; 2015. [Acesso 12 jan 2018]. Disponível em: https://goo.gl/ mXBprD

16. Teixeira MB, Ramôa ML, Ergstron E, Ribeiro JM. Tensions between approach paradigms in public policies 
on drugs: an analysis of Brazilian legislation in 2000-2016. Ciênc Saúde Coletiva. 2017];22(5):1455-66. doi: http:// dx.doi.org/10.1590/1413-81232017225.32772016

17. Méndez E. Uma Versão Brasileira do AUDIT (Alcohol Use Disorders Identification Test). [Internet]. Pelotas: Universidade Federal de Pelotas; 1999. [Acesso 17 set 2020]. Disponível em: https://goo.gl/cYEQby

18. Lima CT, Freire ACC, Silva APB, Teixeira RM, Farrel $M$, Prince $M$. Concurrent and construct validity of the AUDIT in an urban Brazilian sample. Alcohol Alcohol. 2005 Dec;40(6):584-9. doi: http://dx.doi.org/10.1093/alcalc/ agh202

19. Pelicioli M, Barelli C, Gonçalves CBC, Hahn SR, Scherer JI. Alcohol consumption and episodic heavy drinking among undergraduate students from the health area of a Brazilian university. J Bras Psiquiatr. 2017;66(3):1506. doi: http://dx.doi.org/10.1590/0047-2085000000164 20. Cardoso FM, Barbosa HA, Costa FM, Vieira MA, Cadeira AP. Factors associated with practice of binge drinking among students of health. Rev CEFAC. 2015 mar-abr;17(2):475-84. doi: http://dx.doi. org/10.1590/1982-021620158914

21. Ferraz L, Rebelatto SL, Schneider GC, Anzolin V. The use of alcohol and tobacco among students of a university in Southern Brazil. Rev Bras Promoção Saúde. 2017;30(1):79-85. doi: http://dx.doi. org/10.5020/18061230.2017.p79

22. Priotto EMTP, Lizzi EAS, Nihei OK. Use of alcohol and other drugs by college students in a border region of southern Brazil. Espaço Saúde. [Internet]. 2015;16(4):719. [cited 2018 May 27]. Available in: http://www.uel. $\mathrm{br} /$ revistas/uel/index.php/espacoparasaude/article/ download/21564/4

23. Parente EA, Ferreira GE, Almeida BC, Alencar JIP Filho, Souza JN, Lima LWO, et al. Alcohol use among medical students: a possible risk for future doctors? ] Health Biol Sci. [Internet]. 2017;5(4):311-9. [cited 2018 May 27]. doi: http://dx.doi.org/10.12662/2317-3076jhbs. v5i4.1224.p311-319.2017

24. Mejía CR, Vargas M, Talledo-Ulfe L, Rodríguez CAS, Benites-López KE, Payano-Chumacero K, et al. Factores asociados a la dependencia de alcohol y tabaco en estudiantes de ocho facultades de medicina peruanas, 2015. Rev Chil Neuro-Psiquiatr. 2016 Dic;54(4):291-8. doi: http://dx.doi.org/10.4067/S0717-92272016000400004 25. Romera LA, Martins RA, Freitas HH, Tinoco DS, Rondina RC. Tempo livre e uso de álcool e outras drogas: estudo comparativo entre estudantes universitários do Brasil e Portugal. Movimento (Porto Alegre). 2018;24(3):765-76. doi: https://doi.org/10.22456/1982-8918.81951

26. Tavolacci MP, Boerg E, Richard L, Meyrignac G, Dechelotte $P$, Ladner J. Prevalence of binge drinking and associated behaviours among 3286 college students in
France. BMC Public Health. 2016 Feb;16:178. doi: https:// doi.org/10.1186/s12889-016-2863-x

27. Bedendo A, Andrade ALM, Opaleye ES, Noto AR. Binge drinking: a pattern associated with a risk of problems of alcohol use among university students. Rev. Latino-Am. Enfermagem. 2017;25:e2925. doi: http://dx.doi. org/10.1590/1518-8345.1891.2925

28. Pinho MC, Souza RCF, Portugal FB, Siqueira MM. Use of alcohol and tobacco among university students of Occupational Therapy at a public university. SMAD, Rev Eletrônica Saúde Mental Álcool Drog. 2020;16(1):112. doi: https://dx.doi.org/10.11606/issn.1806-6976. smad.2020.152411

29. Fernandes MA, Moita FK, Nascimento MPV, Santos JDM, Paiva MHP. Alcohol consumption among nursing students of a university center. SMAD, Rev Eletrônica Saúde Mental Álcool Drog. 2019;15(2):38-44. doi: https:// dx.doi.org/10.11606/issn.1806-6976.smad.2019.000401 30. Araujo CM, Vieira CX, Mascarenhas CHM. Prevalence of licit and illicit drugs by college students. SMAD, Rev Eletrônica Saúde Mental Álcool Drog. 2018;14(3):14450. doi: https://doi.org/10.11606/issn.1806-6976. smad.2018.000342

31. Carneiro ALM, Rodrigues SB, Gherardi-Donato ECS, Guimarães EAA, Oliveira VC. Padrão do uso de álcool entre estudantes universitários da área da saúde. RECOM. 2014 jan/abr;4(1):940-50. doi: http://dx.doi.org/10.19175/ recom.v0i0.449

32. Damasceno RO, Boery RNSO, Ribeiro IJS, Anjos KF, Santos VC, Boery EN. Uso de álcool, tabaco e outras drogas e qualidade de vida de estudantes universitários Rev Baiana Enferm. 2016 jul/set;30(3):1-10. doi: http:// dx.doi.org/10.18471/rbe.v30i3.15533

33. Dantas LR, Gomes MC, Lima LCM, Cruz-daSilva BR, Dantas LR, Granville-Garcia AF. Use of psychoactive substances at least once in life among Brazilian university students at the beginning and end of courses and the associated factors. Cad Saúde Coletiva. 2017 Dec;25(4):468-74. doi: http://dx.doi. org/10.1590/1414-462×201700040091

34. Souza J, Ornella KP, Almeida LY, Domingos SGA, Andrade LS, Zanetti ACG. Drug use and knowledge of its consequences among nursing students. Texto Contexto Enferm. 2018;27(2):e5540016. doi: https:// doi.org/10.1590/0104-070720180005540016

\section{Contribuição dos autores}

Concepção e planejamento do estudo: Bárbara Souza de Medeiros Nunes, Guilherme Correa Barbosa, Thiago da Silva Domingos. Obtenção dos dados: Thiago da Silva Domingos. Análise e interpretação dos dados: Bárbara Souza de Medeiros Nunes, Guilherme Correa Barbosa, Thiago da Silva Domingos. Análise estatística: Bárbara Souza de Medeiros Nunes, Guilherme Correa Barbosa, 
Thiago da Silva Domingos. Obtenção de financiamento: Bárbara Souza de Medeiros Nunes, Guilherme Correa Barbosa, Thiago da Silva Domingos. Redação do manuscrito: Bárbara Souza de Medeiros Nunes, Guilherme Correa Barbosa, Thiago da Silva Domingos. Revisão crítica do manuscrito: Guilherme Correa Barbosa, Thiago da Silva Domingos.

Todos os autores aprovaram a versão final do texto.

Conflito de interesse: os autores declararam que não há conflito de interesse.

Esta licença permite que outros distribuam, remixem, adaptem e criem a partir do seu trabalho, mesmo para fins comerciais, desde que the atribuam o devido crédito pela criação original. É a licença mais flexível de todas as licenças disponíveis. É recomendada para maximizar a disseminação e uso dos materiais licenciados. 